\title{
Evaluation of a Modified Coagulation and Clipping Method To Prevent Postoperative Bleeding After Gastric Endoscopic Submucosal Dissection: A Multicenter Study
}

Kei Terasaki ( $\nabla$ t-kei@koto.kpu-m.ac.jp )

Saiseikai Suita Hospital

Kotaro Okuda

Saiseikai Suita Hospital

Kyoko Sakai

Saiseikai Suita Hospital

Takao Shirono

Saiseikai Suita Hospital

Shohei Amioka

Saiseikai Suita Hospital

Takahiro Nonaka

Saiseikai Suita Hospital

Takahiro Miura

Saiseikai Suita Hospital

Naoto Iwai

Fukuchiyama City Hospital

Seiji Fujino

Kyoto Kujo Hospital

Hirohisa Oya

Saiseikai Suita Hospital

Takayuki Katayama

Saiseikai Suita Hospital

Kiichirou Miyawaki

Kyoto Kujo Hospital

Takashi Okuda

Fukuchiyama City Hospital

Junko Matsumoto

Saiseikai Suita Hospital

Yasuhide Mitsumoto

Saiseikai Suita Hospital 


\section{Chiemi Mizuno}

Saiseikai Suita Hospital

Masayuki Mizuno

Saiseikai Suita Hospital

Shoji Mitsufuji

Kyoto Kujo Hospital

Toshihide Shima

Saiseikai Suita Hospital

\section{Yoshito Itoh}

Kyoto Prefectural University of Medicine

\section{Takeshi Okanoue}

Saiseikai Suita Hospital

\section{Research Article}

Keywords: Blood Coagulation, Endoscopic Mucosal Resection, Multivariate Analysis, Postoperative Blood Loss, Propensity Score

Posted Date: January 27th, 2022

DOI: https://doi.org/10.21203/rs.3.rs-1282799/v1

License: (c) (i) This work is licensed under a Creative Commons Attribution 4.0 International License. Read Full License 


\section{Abstract}

Postoperative bleeding is one of the most frequent adverse events in endoscopic submucosal dissection (ESD). Post-ESD coagulation (PEC) is widely performed to prevent postoperative bleeding; nonetheless, the postoperative bleeding rate after gastric ESD remains $3.1-5.5 \%$. This study aimed to compare postoperative bleeding rates between patients receiving conventional treatment and those receiving the modified coagulation and clipping (MCC) treatment. Overall, 481 patients were treated between April 2007 and July 2020. The PEC method was perfomed from April 2007 to June 2010, whereas the MCC method was performed from July 2010 to July 2020. Clinicopathological characteristics were retrospectively compared between patients who underwent these two methods using univariate analysis and propensity score matching (PSM) analysis. Of the 481 included patients, 160 and 321 underwent PEC and MCC, respectively. Nine patients (5.6\%) in the PEC group developed postoperative bleeding, whereas no patient $(0.0 \%)$ in the MCC group developed postoperative bleeding. The univariate analysis and PSM analysis showed that the MCC method was significantly associated with the prevention of postoperative bleeding. In conclusion, the postoperative bleeding rate is lower with the MCC method than with the conventional method. The MCC method may be beneficial in preventing postoperative bleeding.

\section{Introduction}

Endoscopic submucosal dissection (ESD) is a minimally invasive treatment for achieving curative resection for early gastric cancer with almost no risk of lymph node metastasis ${ }^{[1-5]}$. However, ESD is still associated with procedure-related adverse events. In particular, postoperative bleeding is a major adverse event in ESD. Postoperative bleeding may cause serious conditions, such as shock, that require transfusion and therefore should be prevented as much as possible.

Hemostatic procedures, such as post-ESD coagulation (PEC) ${ }^{[6]}$ are performed for post-ESD ulcers to

prevent postoperative bleeding, which occurs in 3.1- 5.5\% of cases ${ }^{[6-8]}$. Mukai et al. ${ }^{[9]}$ and Azumi et al. ${ }^{[10]}$ reported the usefulness of coagulation plus artery-selective clipping (2C) and search, coagulation, and clipping (SCC), respectively. These procedures reduced the postoperative bleeding rate compared with PEC alone ${ }^{[9,10]}$. We further modified this method of coagulation and clipping to reduce the risk of postoperative bleeding. Our approach requires thorough coagulation of arteries and residual vessels at the margin of the ESD ulcer. Additional clipping involving the muscular layers is performed to provide additional strength and prevent delayed perforation due to overcoagulation. This modified coagulation and clipping (MCC) method was termed the "MCC method".

This study aimed to compare postoperative bleeding rates between patients receiving conventional treatment and those who underwent the MCC method.

\section{Results}


A total of 481 (349 male and 132 female) patients were enrolled in this study. The clinicopathological characteristics of these patients are summarized in Table 1 . The mean age was $71.1 \pm 10.0$ years. Out of 481 patients, 89 (18.5\%) were on antithrombotic therapy for their comorbidities.

Furthermore, out of the 481 patients, 160 and 321 underwent PEC and the MCC method, respectively. Table 2 shows the clinicopathological characteristics of the two groups. There were significant differences in tumor size $(p=0.034)$, resected specimen size $(p=0.019)$, operation time $(p<0.001)$, and postoperative bleeding $(p<0.001)$ between the two groups. In total, nine patients developed postoperative bleeding; however, all of these patients were in the PEC group, with no patient in the MCC group. All cases of postoperative bleeding were successfully treated by endoscopic hemostasis. No major hemorrhage occurred.

Next, we compared the incidence of postoperative bleeding in patients on antithrombotic therapy between the two groups. Twenty-five patients (15.6\%) in the PEC group and 64 patients (19.9\%) in the MCC group were on antithrombotic therapy. Postoperative bleeding occurred in $4 \%(1 / 25)$ and $0 \%(0 / 64)$ of patients in the PEC and MCC groups, respectively. No significant difference was observed between the two groups $(p=0.281)$, although the incidence of postoperative bleeding was lower in the MCC group than in the PEC group (Table3).

We performed a quasi-randomized comparison with propensity score matching (PSM) to reduce the selection bias between the two groups. After PSM, there were 143 matched pairs of patients between the two groups. Table 4 presents the details of the propensity score-matched patients. A significant difference in postoperative bleeding was identified between the two groups $(p=0.007)$.

\section{Discussion}

We investigated the usefulness of the MCC method in preventing postoperative bleeding after gastric ESD. Postoperative bleeding after gastric ESD requires invasive intervention and, to date, has been difficult to eliminate.

Mukai et al. ${ }^{[9]}$ and Azumi et al. ${ }^{[10]}$ reported that the $2 \mathrm{C}$ and SCC methods, respectively, reduced the postoperative bleeding rate compared with PEC alone. In this study, no patient in the MCC group developed postoperative bleeding after gastric ESD. There were no complications associated with the MCC method. These results suggest that additional clipping after PEC may be useful in preventing postoperative bleeding after gastric ESD. The main difference between the MCC method and the other two clipping methods is the application of coagulation and clipping. The $2 \mathrm{C}$ and SCC methods coagulate vessels and clip for additional strength. In contrast, the MCC method coagulates vessels thoroughly and applies clips not only to provide additional strength but also to prevent delayed perforation due to overcoagulation. After high-risk vessels and the residual vessels at the margin of the ESD ulcer were thoroughly coagulated, additional clipping involving the muscular layers surrounding the vessels was performed with sufficient clips. Accordingly, more clips are used in the MCC method (mean, 7.9 clips) 
than in either of the other two methods (mean, 3.8-4.8 clips). These factors may aid in preventing postoperative bleeding after gastric ESD.

In an aging society, the number of patients receiving antithrombotic therapy and those undergoing ESD has increased. Antithrombotic therapy is a risk factor for postoperative bleeding, particularly in patients receiving dual antiplatelet agents and heparin replacement therapy ${ }^{[11-15]}$ after the publication of the Japan Gastroenterological Endoscopy Society guidelines (JGES) ${ }^{[16]}$. Although 64 patients $(19.9 \%)$ in the MCC group underwent gastric ESD while receiving antithrombotic therapy and most cases were reported after the guidelines were established, there were no cases of postoperative bleeding. Therefore, these findings suggest that the MCC method may also be useful in preventing postoperative bleeding in patients receiving antithrombotic therapy.

From an economic point of view, one hemostatic clip costs 7.5 USD/825 JPY. Mukai et al. ${ }^{\left[{ }^{[9}\right.}$ and Azumi et al. ${ }^{[10]}$ reported that 3.8 and 4.9 clips were required on average, respectively. In this study, we used 7.9 clips on average. Thus, the cost was 59.2 USD/6,517.5 JPY. Considering that the incidence of postoperative bleeding is approximately $5 \%$, it may be considered excessive to perform the MCC method for all patients undergoing gastric ESD. However, if postoperative bleeding occurs, a drastic situation may unfold. Therefore, the MCC procedure may be a treatment option, at least for patients at a high risk of postoperative bleeding, such as those receiving antithrombotic therapy.

This study had some limitations. First, this study was performed in three institutions with a limited number of patients. Second, this study had a retrospective design. Therefore, PSM was performed to minimize differences between the PEC and MCC groups. Third, the period when ESD was performed differed between the PEC and MCC groups. Thus, technical improvements in ESD might have influenced the outcomes.

In conclusion, this study suggests that the postoperative bleeding rate was lower with the MCC method than with the conventional method. Therefore, the MCC method may be beneficial in preventing postoperative bleeding. The efficacy of the MCC method must be further confirmed by a randomized controlled trial.

\section{Methods}

\section{Patients}

A total of 481 patients who underwent gastric ESD for gastric cancer at Fukuchiyama City Hospital, Kyoto Kujo Hospital and Saiseikai Suita Hospital between April 2007 and July 2020 were enrolled. In patients who underwent ESD for two or more lesions, the largest lesion was selected as the representative lesion. The protocol for this research project has been approved by the Clinical Ethics Committees on Human Experiments of Fukuchiyama City Hospital, Kujo Hospital and Saiseikai Suita Hospital, and it conforms to the provisions of the Declaration of Helsinki (as revised in Fortaleza, Brazil, October 2013). Informed consent was obtained from all patients before participation in the study. 


\section{Patients' characteristics}

We reviewed patients' medical records and collected the following data: age, sex, antithrombotic agents, tumor location, tumor size, resected specimen size, histology, invasion depth, presence of ulceration, operation time, number of clips used, time of clipping, and ESD complications.

\section{Study Design}

This was a multicenter retrospective cohort study. Between April 2007 and June 2010, PEC was performed after gastric ESD. Between July 2010 and July 2020, MCC was performed after gastric ESD. We assessed the incidence of postoperative bleeding after gastric ESD for each of these methods.

\section{ESD procedure and management after ESD}

Gastric ESD was performed with an insulated-tip knife-2 (KD-611L, Olympus, Tokyo, Japan), a Flush knife (DK2620J; Fujifilm, Tokyo, Japan) and a clutch cutter (DP2618DT; Fujifilm, Tokyo, Japan) through a conventional single-channel endoscope (EG-580RD; Fujifilm, Tokyo, Japan and Q260J; Olympus, Tokyo, Japan). Marker dots were made approximately $5 \mathrm{~mm}$ from the lesion. After submucosal injection of hyaluronic acid, a circumferential mucosal incision was made outside the marked region. Submucosal dissection was performed in all cases. Hemostasis of active bleeding and preventive coagulation of all visible vessels were performed during or after ESD using hemostatic forceps (FD-411QR; Coagrasper, Olympus, Tokyo, Japan) or hot biopsy forceps (Hoya Co. Ltd., Pentax Life Care Div, Tokyo, Japan).

A proton-pump inhibitor was intravenously injected twice daily from the day before ESD to the day after ESD. Patients were supplied with drinking water $2 \mathrm{~h}$ after ESD. A second-look endoscopy was performed the next day. Liquid food was also provided with no complications. Oral proton-pump inhibitors were administered from 2 days after ESD to 8 weeks later.

\section{Management of antithrombotic agents}

For patients receiving oral antithrombotic agents, such as low-dose aspirin, thienopyridine, cilostazol, warfarin, dabigatran, rivaroxaban, apixaban, and edoxaban, the prescribing doctor was consulted regarding the management of antithrombotic agents before ESD. Before the publication of the JGES guidelines for gastroenterological endoscopy in patients undergoing antithrombotic treatment, antithrombotic agents were almost always discontinued before ESD. After the publication of the JGES guidelines, the management of antithrombotic agents was determined according to the guidelines.

\section{MCC method}

For patients undergoing the MCC method, the operator or assistant recorded the location of the blood vessels that caused intraoperative bleeding and required hemostasis with hemostatic forceps or in which preventive coagulation with hemostatic forceps was performed. 
After the ESD procedure, additional coagulation was performed thoroughly at the sites where the location was recorded, at the penetrating blood vessels in the muscular layer, and at the margin of the ESD ulcer. Additional hemostatic clips (HX-610-135; Olympus, Tokyo, Japan) were applied to include the muscular layer for additional strength of the vessels and to prevent delayed perforation owing to overcoagulation (Figs 1 and 2).

\section{Definition of postoperative bleeding}

Postoperative bleeding was defined as an event requiring urgent endoscopic hemostasis after gastric ESD, such as hematemesis, melena, or a decrease in hemoglobin concentration by $>2 \mathrm{~g} / \mathrm{dl}$. Endoscopic hemostasis was performed when active bleeding or blood coagulation was observed. Preventive hemostasis of visible vessels without bleeding was not regarded as postoperative bleeding.

\section{Statistical analysis}

For descriptive statistics, continuous variables are presented as mean \pm standard deviation or median (range), whereas discrete variables are presented as frequency and proportion. All statistical analyses were performed using STATA version 12.1 (Stata Corp LLC 4905 Lakeway Drive College Station, Texas 77845 USA) and EZR (Saitama Medical Center, Jichi Medical University, Saitama, Japan), R version 2.13.0 (The R Foundation for Statistical Computing, Vienna, Austria), with $p$-values $<0.05$ being considered statistically significant.

\section{Propensity score}

To reduce the effect of procedure-selection bias, we performed PSM of MCC procedure selection. The scores were calculated as the log-odds obtained by the logistic regression model, with response variables including the PEC or MCC groups. Explanatory variables consisted of tumor location, tumor size, resected specimen size, invasion depth, histological types, antithrombotic agents, procedure time. Matching was performed using a 1:1 matching protocol with nearest-neighbor matching within a caliper width of 0.01 without replacement. We used the receiver operating characteristic and area under the curve to measure the balance of covariates. The matched data sets were checked for balance in terms of an absolute standardized difference. The propensity score was rounded from three to two decimal places. Matching was performed using three decimal places for the PEC and MCC groups.

After PSM, we compared the postoperative bleeding rates between the PEC and MCC groups. A twosided $p$-value of $<0.05$ in the chi square test or Fisher's exact test was considered statistically significant. PSM was performed using EZR (Saitama Medical Center, Jichi Medical University, Saitama, Japan).

\section{Declarations}

Acknowledgments 
We thank all members of the Department of Gastroenterology and Hepatology, Saiseikai Suita Hospital; the Department of Laboratory Medicine, Saiseikai Suita Hospital; the Department of Molecular Gastroenterology and Hepatology, Graduate School of Medical Science, Kyoto Prefectural University of Medicine; the Department of Gastroenterology, Kyoto Kujo Hospital; and the Department of Gastroenterology and Hepatology, Fukuchiyama City Hospital for helping us in performing this study. We also thank Editage (www.editage.jp) for the English language review.

\section{Author contributions}

K.T. performed the research, analyzed the data, and wrote the manuscript; K.O. designed the research study. K.S. analyzed the data. All other authors contributed to the collection and interpretation of data for the work, and critically reviewed the manuscript. All authors have approved the final version of the manuscript and agree to be accountable for all aspects of the work in ensuring that questions related to the accuracy or integrity of any part of the work are appropriately investigated and resolved.

\section{Data availability statement}

The data that support the findings of this study are available from the corresponding author upon reasonable request.

Competing interests: Drs. Kei Terasaki, Kotaro Okuda, Kyoko Sakai, Takao Shirono, Shohei Amioka, Takahiro Nonaka, Takahiro Miura, Naoto Iwai, Seiji Fujino, Hirohisa Oya, Takayuki Katayama, Kiichirou Miyawaki, Takashi Okuda, Junko Matsumoto, Yasuhide Mitsumoto, Chiemi Mizuno, Masayuki Mizuno, Shoji Mitsufuji, Toshihide Shima, Yoshito Itoh, and Takeshi Okanoue have no conflicts of interest or financial ties to disclose.

Funding Information: This research did not receive any specific grant from funding agencies in the public, commercial, or not-for-profit sectors.

\section{References}

1. Abe, N. et al. Key factors for successful en bloc endoscopic submucosal dissection of early stage gastric cancer using an insulation-tipped diathermic knife. Hepato-Gastroenterology 53, 639-642 (2006). Pubmed:16995479.

2. Gotoda, T. Endoscopic resection of early gastric cancer. Gastric Cancer 10, 1-11 (2007). 10.1007/s10120-006-0408-1.

3. Kim, B. J. et al. Efficacy and safety of endoscopic submucosal dissection for early gastric cancer in patients with comorbid diseases. Gut Liver 4, 186-191 (2010). 10.5009/gnl.2010.4.2.186, Pubmed:20559520.

4. Oda, I. et al. A multicenter retrospective study of endoscopic resection for early gastric cancer. Gastric Cancer 9, 262-270 (2006). 10.1007/s10120-006-0389-0, Pubmed:17235627. 
5. Ono, H. et al. Endoscopic mucosal resection for treatment of early gastric cancer. Gut $48,225-229$ (2001). 10.1136/gut.48.2.225, Pubmed:11156645.

6. Takizawa, K. et al. Routine coagulation of visible vessels may prevent delayed bleeding after endoscopic submucosal dissection - An analysis of risk factors. Endoscopy 40, 179-183 (2008). 10.1055/s-2007-995530, Pubmed:18322872.

7. Oda, I., Suzuki, H., Nonaka, S. \& Yoshinaga, S. Complications of gastric endoscopic submucosal dissection. Dig. Endosc. 25 Supplement 1, 71-78 (2013). 10.1111/j.1443-1661.2012.01376.x, Pubmed:23368986.

8. Okada, K. et al. Risk factors for delayed bleeding after endoscopic submucosal dissection for gastric neoplasm. Surg. Endosc. 25, 98-107 (2011). 10.1007/s00464-010-1137-4, Pubmed:20549245.

9. Mukai, S. et al. Postprocedural combined treatment using the coagulation plus artery-selective clipping (2C) method for the prevention of delayed bleeding after ESD. Surg. Endosc. 27, 1292-1301 (2013). 10.1007/s00464-012-2600-1, Pubmed:23232998.

10. Azumi, M. et al. The search, coagulation, and clipping (SCC) method prevents delayed bleeding after gastric endoscopic submucosal dissection. Gastric Cancer 22, 567-575 (2019). 10.1007/s10120-0180878-y, Pubmed:30267178.

11. Terasaki, K. et al. Effects of guidelines for gastroenterological endoscopy in patients undergoing antithrombotic treatment on postoperative bleeding after endoscopic submucosal dissection for early gastric cancer: A propensity score-matching analysis. Digestion 102, 256-264 (2021). 10.1159/000504597, Pubmed:31770748.

12. Shindo, Y., Matsumoto, S., Miyatani, H., Yoshida, Y. \& Mashima, H. Risk factors for postoperative bleeding after gastric endoscopic submucosal dissection in patients under antithrombotics. World J. Gastrointest. Endosc. 8, 349-356 (2016). 10.4253/wjge.v8.i7.349, Pubmed:27076874.

13. Sanomura, Y. et al. Taking warfarin with heparin replacement and direct oral anticoagulant is a risk factor for bleeding after endoscopic submucosal dissection for early gastric cancer. Digestion 97, 240-249 (2018). 10.1159/000485026, Pubmed:29421806.

14. Igarashi, K. et al. Should antithrombotic therapy be stopped in patients undergoing gastric endoscopic submucosal dissection? Surg. Endosc. 31, 1746-1753 (2017). 10.1007/s00464-0165167-4, Pubmed:27530896.

15. Furuhata, T. et al. Postoperative bleeding after gastric endoscopic submucosal dissection in patients receiving antithrombotic therapy. Gastric Cancer 20, 207-214 (2017). 10.1007/s10120-015-0588-7, Pubmed:26754296.

16. Fujimoto, K. et al. Guidelines for gastroenterological endoscopy in patients undergoing antithrombotic treatment. Dig. Endosc. 26, 1-14 (2014). 10.1111/den.12183, Pubmed:24215155.

\section{Tables}


Table 1. Clinical characteristics of all patients and gastric lesions

\begin{tabular}{ll}
\hline Variable & All patients $(\mathrm{n}=481)$ \\
\hline Sex (male/female), $\mathrm{n}(\%)$ & $349 / 132(72.6 / 27.4)$ \\
\hline Age (mean \pm SD) (year) & $71.1 \pm 10.0$ \\
\hline Antithrombotic agent, $\mathrm{n}(\%)$ & $89(18.5)$ \\
\hline Tumor location, $\mathrm{n}(\%)$ & $74(15.4)$ \\
\hline \multicolumn{1}{c}{ Upper third of the stomach } & $133(27.7)$ \\
\hline \multicolumn{1}{|c}{ Middle third of the stomach } & $274(56.9)$ \\
\hline \multicolumn{1}{c}{ Lower third of the stomach } & $14.7 \pm 10.3$ \\
\hline Tumor size (mean \pm SD) (mm) & $35.9 \pm 12.7$ \\
\hline Resected specimen size (mean \pm SD) (mm) & \\
\hline Histological type of the tumor, $\mathrm{n}(\%)$ & $465(96.7)$ \\
\hline Differentiated & $16(3.3)$ \\
\hline Undifferentiated & \\
\hline Invasion depth, $\mathrm{n}(\%)$ & $438(91.1)$ \\
\hline T1a & $43(8.9)$ \\
\hline T1b or more & $37(7.7)$ \\
\hline Presence of ulceration, $\mathrm{n}(\%)$ & $75.7 \pm 72.9$ \\
\hline Operation time (mean \pm SD) (min) & \\
\hline Abbreviation: SD, standard deviation &
\end{tabular}


Table 2. Clinicopathological characteristics of patients in the PEC and MCC groups

\begin{tabular}{|c|c|c|c|}
\hline Variable & PEC group & MCC group & $p$ value \\
\hline & $(n=160)$ & $(n=321)$ & \\
\hline Sex (male/female), n (\%) & $123 / 37(76.9 / 23.1)$ & $226 / 95(70.4 / 29.6)$ & 0.159 \\
\hline Age (mean \pm SD) (year) & $70.1 \pm 10.2$ & $71.3 \pm 9.9$ & 0.859 \\
\hline Antithrombotic agent, n (\%) & $25(15.6)$ & $64(19.9)$ & 0.264 \\
\hline Heparin replacement therapyt, $\mathrm{n}(\%)$ & $8(5.0)$ & $9(2.8)$ & 0.222 \\
\hline Tumor location, $\mathrm{n}(\%)$ & & & 0.404 \\
\hline Upper third of the stomach & $29(18.0)$ & $45(14.0)$ & \\
\hline Middle third of the stomach & $46(28.7)$ & $87(27.1)$ & \\
\hline Lower third of the stomach & $85(53.1)$ & $189(58.9)$ & \\
\hline Tumor size $($ mean $\pm \mathrm{SD})(\mathrm{mm})$ & $15.7 \pm 9.8$ & $14.2 \pm 10.5$ & 0.034 \\
\hline Resected specimen size (mean $\pm \mathrm{SD})(\mathrm{mm})$ & $37.8 \pm 13.0$ & $34.9 \pm 12.4$ & 0.019 \\
\hline Histological type of the tumors, $n(\%)$ & & & 0.103 \\
\hline Differentiated & $158(98.8)$ & $307(95.6)$ & \\
\hline Undifferentiated & $2(1.2)$ & $14(4.4)$ & \\
\hline Invasion depth, n (\%) & & & 0.127 \\
\hline T1a & $141(88.1)$ & $297(92.5)$ & \\
\hline T1b or more & $19(11.9)$ & $24(7.5)$ & \\
\hline Presence of ulceration, $\mathrm{n}(\%)$ & $12(7.5)$ & $25(7.8)$ & 1.000 \\
\hline Operation time (mean $\pm \mathrm{SD})(\mathrm{min})$ & $101.6 \pm 100.1$ & $62.8 \pm 49.8$ & $<0.001$ \\
\hline Postoperative bleeding, n (\%) & $9(5.6)$ & $0(0)$ & $<0.001$ \\
\hline Number of clips (mean \pm SD) & 0 & $7.9 \pm 4.4$ & \\
\hline Time of clipping (mean \pm SD) & 0 & $13.5 \pm 9.2$ & \\
\hline Delayed perforation, $\mathrm{n}(\%)$ & $2(1.3)$ & $0(0)$ & 0.110 \\
\hline
\end{tabular}

Abbreviations: MCC, modified coagulation and clipping;

PEC, post-endoscopic submucosal dissection coagulation; SD, standard deviation. 
Table 3. Clinicopathological characteristics of patients on antithrombotic therapy in the PEC and MCC groups

\begin{tabular}{llll}
\hline Characteristic & PEC group & MCC group & $p$ value \\
\hline Postoperative bleeding, $\mathrm{n}(\%)$ & $(\mathrm{n}=25)$ & $(\mathrm{n}=64)$ & \\
\hline Antithrombotic agent, $\mathrm{n}(\%)$ & $1(4)$ & $0(0)$ & 0.281 \\
\hline Single antiplatelet agent & $16(64)$ & $46(71.8)$ & 0.197 \\
\hline Dual antiplatelet agents & $2(8)$ & $4(6.3)$ & 1.000 \\
\hline Anticoagulant agents & $7(28)$ & $14(21.9)$ & 1.000 \\
\hline Heparin replacement therapy, $\mathrm{n}(\%)$ & $8(32)$ & $9(14.1)$ & 0.293 \\
\hline
\end{tabular}

Abbreviations: MCC, modified coagulation and clipping;

PEC, post-endoscopic submucosal dissection coagulation. 
Table 4. Clinicopathological characteristics of patients in the PEC and MCC groups after propensity score matching

Variable

\begin{tabular}{|c|c|c|c|c|}
\hline & PEC method & MCC method & ASD & $\begin{array}{l}P \\
\text { value }\end{array}$ \\
\hline & $(n=143)$ & $(n=143)$ & & \\
\hline Sex (Male/Female), n (\%) & $\begin{array}{l}109 / 34 \\
(76.2 / 23.8)\end{array}$ & $\begin{array}{l}100 / 43 \\
(69.9 / 30.1)\end{array}$ & 0.14 & 0.286 \\
\hline Age (mean $\pm S D$ ),year & $71.6 \pm 9.6$ & $71.9 \pm 9.5$ & 0.03 & 0.686 \\
\hline Antithrombotic agent, n (\%) & $23(16.1)$ & $26(18.2)$ & 0.05 & 0.754 \\
\hline Heparin replacement therapy, $\mathrm{n}$ & 8 & 4 & & 0.380 \\
\hline Tumor location, n (\%) & & & 0.00 & 0.683 \\
\hline Upper third of the stomach & $23(16.1)$ & $19(13.3)$ & & \\
\hline Middle third of the stomach & $41(28.7)$ & $47(32.9)$ & & \\
\hline Lower third of the stomach & $79(55.2)$ & $77(53.8)$ & & \\
\hline Tumor size (mean $\pm \mathrm{SD}), \mathrm{mm}$ & $15.1 \pm 9.6$ & $15.6 \pm 12.1$ & 0.04 & 0.716 \\
\hline $\begin{array}{l}\text { Resected specimen size (mean } \pm \\
\text { SD),mm }\end{array}$ & $36.4 \pm 12.4$ & $36.2 \pm 12.8$ & 0.02 & 0.870 \\
\hline Histological type of the tumors, $\mathrm{n}(\%)$ & & & 0.14 & 0.498 \\
\hline Differentiated & $141(98.6)$ & $143(100)$ & & \\
\hline Undifferentiated & $2(1.4)$ & $0(0)$ & & \\
\hline Invasion depth, n (\%) & & & 0.13 & 0.441 \\
\hline T1a & $133(93.0)$ & $137(95.8)$ & & \\
\hline T1b or more & $10(7.0)$ & $6(4.2)$ & & \\
\hline Presence of ulceration, n (\%) & 11 & 16 & & 0.419 \\
\hline Postoperative bleeding, n (\%) & $8(5.6)$ & $0(0)$ & & 0.007 \\
\hline Operation time $($ mean $\pm S D)(\min )$ & $78.1 \pm 66.1$ & $77.2 \pm 64.3$ & 0.01 & 0.908 \\
\hline Number of clips (mean \pm SD) & 0 & $8.6 \pm 4.4$ & & \\
\hline Time of clipping (mean \pm SD) & 0 & $14.3 \pm 9.3$ & & \\
\hline Delayed perforation, $\mathrm{n}(\%)$ & $2(1.4)$ & $0(0)$ & & 0.500 \\
\hline
\end{tabular}

Abbreviations: MCC, modified coagulation and clipping; PEC, post-endoscopic submucosal dissection 
coagulation;

SD, standard deviation.

\section{Figures}

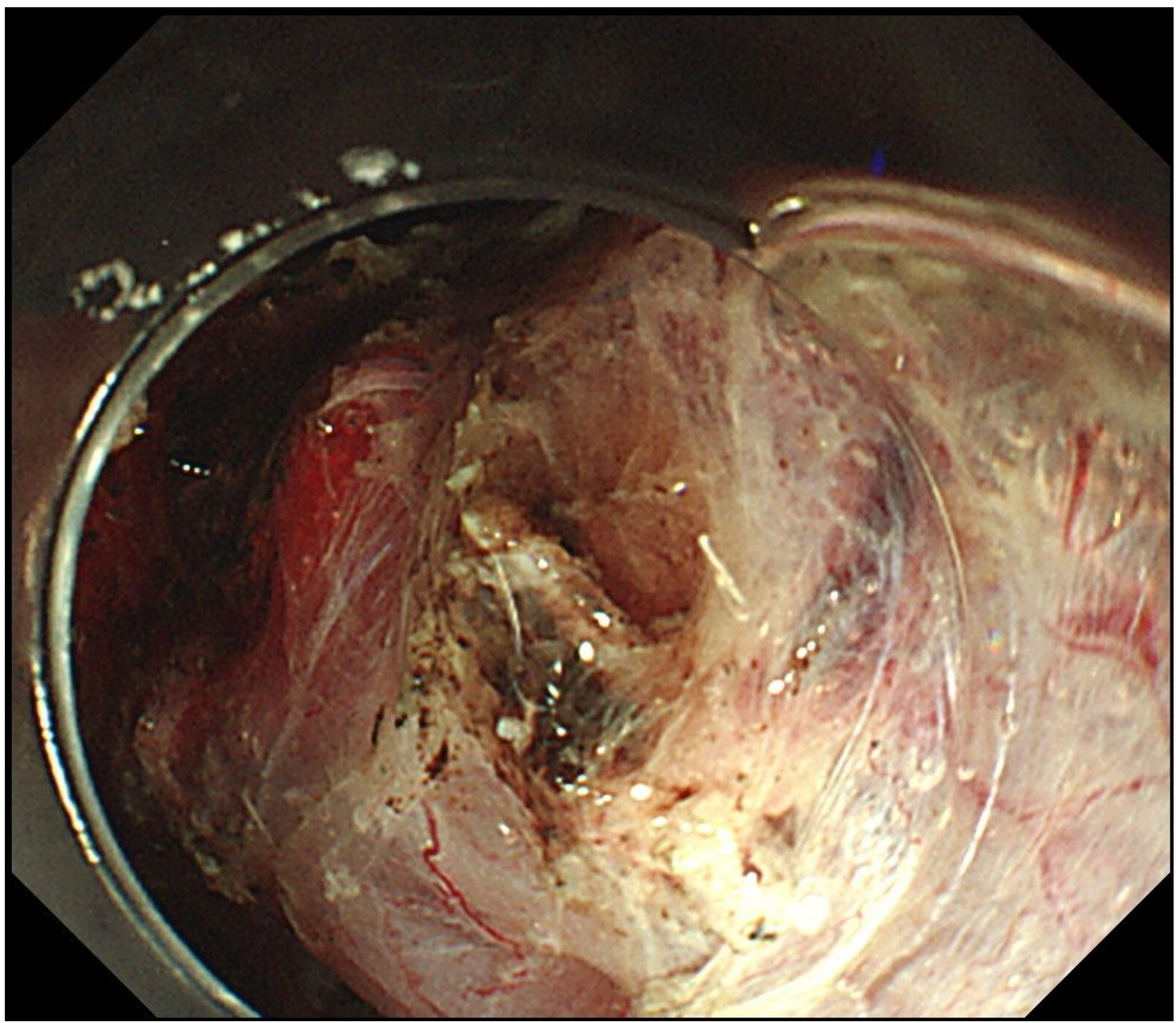

\section{Figure 1}

The blood vessel with pulsation was detected. Thus, coagulation using hemostatic forceps was performed, and hemostatic clips were applied. 


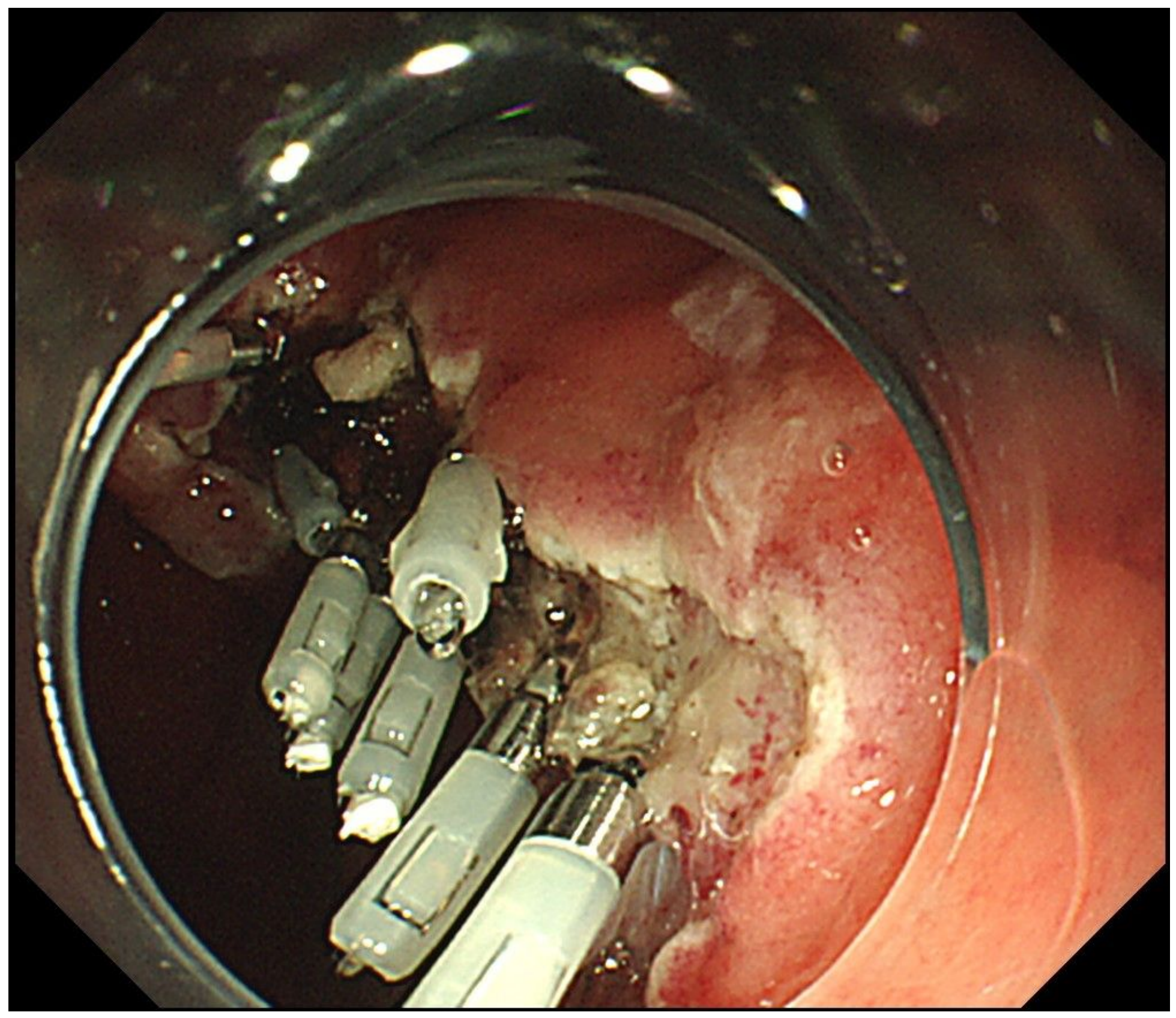

Figure 2

Post-ESD ulcer after MCC method was performed. MCC, modified coagulation and clipping. 